\title{
Die Zerstörung der Phosphore durch positive Strahlen.
}

\author{
Von G. Berndt.
}

(Eingegangen am 19. Dezember 1919.)

Bei den radioaktiven Leuchtfarben (welche aus einem Gemisch von Sidotblende und einem oder mehreren Alphastrahlern bestehen) beobachtet man, auch bei konstanter Radioaktivität, ein allmähliches Nächlassen der Leuchtkraft ${ }^{1}$ ), welches von Rutherford ${ }^{2}$ ) auf eine Zerstörung der einmal durch ein Alphateilchen getroffenen, allein erregbaren komplexen Zentren zurückgeführt wird. Auf Grund dieser Annahme hat er für die Abnahme der Intensität $J$ (bei konstanter Strahlung) mit der Zeit $t$ die Gleichung abgeleitet

$$
J / J_{0}=\frac{1}{A \cdot t}\left(1-e^{-A \cdot t}\right)
$$

in welcher $J_{\theta}$ die Anfangshelligkeit bedeutet und

$$
A=K \cdot P \cdot q / N
$$

ist, wo $P$ die Zahl der in einer Sekunde auftreffenden Teilchen, $q$ die von einem Teilchen erzeugte $Z$ ahl von Ionen und $N$ die Zahl der Phosphormoleküle im Kubikzentimeter ist. Dabei ist vorausgesetzt, daß die Ionisierung $i$ auf der Strecke $D$ (innerhalb der Reichweite $R$ ) nach der Gleichung abnimmt

$$
i / i_{0}=e^{-\boldsymbol{K} \cdot D} \text {; }
$$

die beste Ubereinstimmung mit der Erfahrung ergab sich für $K=4,5 / R$. Die Gleichung (1) stellt die Beobachtungen von Marsden ${ }^{3}$ ) und von Berndt ${ }^{4}$ ) recht gut dar.

Ganz ähnliche Verhältnisse wie bei der Radiolumineszenz liegen nun auch bei der Phosphoreszenzerregung durch Kanalstrahlen vor. Auch hierbei leuchtet die Sidotblende am hellsten von allen bisher untersuchten Phosphoren 5), ferner sind auch von H. v. Dechend und W. Hammer") bei dieser Szintillationen durch Wasserstoff-

1) Literatur bei G. Berndt, Radioaktive Leuchtfarben. Braunsehweig, Friedr. Vieweg \& Bohn, 1920.

2) E. Rutherford, Proc. Roy. Boc. (A) 83, 561, 1910.

3) F. Mars den, Proc. Roy: Soe. (A) 83, 548, 1910.

4) G. Berndt, 1. c.

5) H. Baerwald, Ann. d. Phys. (4) 39, 849, 1912.

6) H. v. Dechend u. W. Hammer, Verh. d. D. Phys. Ges. 12, 531, 1910. 
Kanalstrahlen beobachtet worden, die allerdings von E. Rüchardt ${ }^{1}$ ) noch bestritten werden. Wie bei den Lenchtfarben ${ }^{2}$ ) ist anch die durch Kanalstrahlen erregte Phosphoreszenz angenähert proportional der Zahl der auftreffenden Teilchen ${ }^{3}$ ). Es rührt dies nach E. Rüchardt ${ }^{4}$ ) daher, daß Sättigung (wie bei der Photolumineszenz) nicht eintreten kann, da es nur bei den durch licht erregten Dauerbanden, nicht aber bei dem durch Kathoden- und Kanalstrahlen erzeugten Ultraviolettprozeß möglich ist, die obere Grenze zu erreichen. Vor allem tritt aber auch bei der Kanalstrahlenphosphoreszenz ein beträchtlicher Rückgang, und zwar wegen der großen Intensität der Strahlung schon in sehr kurzer Zeit ein, der bei dem heller lenchtenden Zinksulfid stärker ausgeprägt ist als bei dem schwächer phosphoreszierenden Willemit 5). Die erschöpften Stellen leuchten aber nach Beobachtungen von J. J. Thomson ${ }^{6}$ ) und E. Goldstein ${ }^{7}$ ) noch bei Kathodenbestrahlung und sind nach E. Rüchardt ${ }^{8}$ ) auch photolumineszenzfähig. Nach diesem wird auch der erschöpfte (und gleichzeitig braun verfärbte) Phosphor durch Erhitzung in der Bunsenflamme wieder völlig regeneriert.

Für den Abfall der Helligkeit bei der Kanalbestrahlung liegen leider bisher wenige Messungen vor, nur E. Rüchardt ${ }^{9}$ ) gibt einige Beobachtungen an einem Sr-Bi-Phosphor graphisch wieder und bemerkt dabei, daß das Abklingen nicht exponentiell erfolgt. Aus der Kurve kann man nun die in der Tabelle (zweite Spalte) mitgeteilten Werte von $J / J_{0}$ für die Zeit $t$ in Sekunden entnehmen.

Da die Radio- und die Kanalstrahlenlumineszenz beide durch den Stoß von Teilchen auf eine phosphoreszierende Substanz erzengt werden, so müßte die Rutherfordsche Theorie auch für diese zutreffen. Versucht man die Beobachtungen durch die Gleichung (1) darzustellen, so erhält man für $A$ die in der dritten Spalte wiedergegebenen Werte, aus denen sich im Mittel $A=0,509$ ergibt. Die hiermit nach Gleichung (1) berechneten Werte von $J / J_{0}$ sind in der vierten und ihre Differenzen gegen die beobachteten Werte in der fünften Spalte mitgeteilt. Wie man sieht, ist der größte anftretende

1) E. Rü chardt, Phys. ZS. 20, 473, 1910.

2) G. Berndt, l. c.

3) J. Königsberger u. J. Kutschewski, Ann. d. Phys. (4) 37, 161, 1912.

4) E. Rüchardt, Ann. d. Phys. (4) 48, 838, 1915.

5) J. Königsberger u. J. Kutschewski, Ann. d. Phys. (4) 37, 161, 1912.

6) J. J. Thoms on, Phil. Mag. (6) 20, 752, 1910; Phys. ZS, 11, 1131, 1910.

7) E. Goldstein, Verh. d. D. Phys. Ges. 14, 33, 1912.

8) E. Rücharat, Ann. d. Phys. (4) 45, 1063, 1914.

9) E. Rüehardt, l. c. S. 1072 . 
Abfall der Helligkeit eines Sr-Bi-Phosphors bei Kanalbestrahlung.

\begin{tabular}{c|c|c|c|c}
\hline \hline$t$ & $J / J_{0}$ beob. & $A$ & $J / J_{0}$ ber. & Differenz \\
\hline 0 & 100 & - & 100 & - \\
1 & 80 & 0,466 & 78,4 & $+1,6$ \\
2,3 & 60 & 0,490 & 59,0 & $+1,0$ \\
4 & 40 & 0,551 & 42,5 & $-2,5$ \\
9,5 & 20 & 0,522 & 20,5 & $-0,5$ \\
15 & 12,8 & 0,517 & 12,9 & $-0,1$ \\
20 & 10 & 0,507 & 10,0 & $\pm 0,0$
\end{tabular}

Untersehied nur 2,5; die tobereinstimmung ist also eine sehr gute, zumal wenn man bedenkt, daß die aus der Kurve entnommenen Werte wegen des kleinen Maßstabes mit einer gewissen Ungenanigkeit behaftet sind. Von vornherein war andererseits zu erwarten, daß sie besser als bei der Radiolumineszenz sein dürftè, da bei der Kanalstrahlenerregung die Intensität sofort mit ihrem Höchstwert einsetzt, wäbrend bei den Leuchtfarben infolge der Bildung alphastrahlender Zerfallsprodukte zưnächst ein Anwachsen der Helligkeit erfolgt, welches die Theorie nicht berücksichtigt; der Zeitpunkt, von welchem ab man die in Gleichung (1) auftretende Zeit $t$ zu zählen hat, ist daher-bei diesen einer gewissen Willkür unterworfen.

Zasammenfassung: An den Beobachtungen von E. Rüchardt über das Abklingen eines Sr-Bi-Phosphors bei der Erregung durch Kanalstrahlen wird nachgewiesen; daß dieses durch dieselbe Gleichung dargestellt werden kann, welche von E. Ru therford für das zeitliche Nachlassen der Radiolumineszenz aufgestellt ist.

Berlin-Friedenau, Dezember 1919.

Phys. Labor. d. Opt. Anstalt C. P. Goerz, A.G. 